\title{
Mutational evolution in a lobular breast tumour profiled at single nucleotide resolution
}

\author{
Sohrab P. Shah ${ }^{1,2 *}$, Ryan D. Morin ${ }^{3 *}$, Jaswinder Khattra ${ }^{1}$, Leah Prentice ${ }^{1}$, Trevor Pugh ${ }^{3}$, Angela Burleigh ${ }^{1}$, \\ Allen Delaney ${ }^{3}$, Karen Gelmon ${ }^{4}$, Ryan Guliany ${ }^{1}$, Janine Senz ${ }^{2}$, Christian Steidl ${ }^{2,5}$, Robert A. Holt ${ }^{3}$, Steven Jones ${ }^{3}$, \\ Mark Sun ${ }^{1}$, Gillian Leung ${ }^{1}$, Richard Moore ${ }^{3}$, Tesa Severson ${ }^{3}$, Greg A. Taylor ${ }^{3}$, Andrew E. Teschendorff ${ }^{6}$, Kane Tse $^{1}$, \\ Gulisa Turashvili ${ }^{1}$, Richard Varhol ${ }^{3}$, René L. Warren ${ }^{3}$, Peter Watson ${ }^{7}$, Yongjun Zhao ${ }^{3}$, Carlos Caldas $^{6}$, \\ David Huntsman ${ }^{2,5}$, Martin Hirst ${ }^{3}$, Marco A. Marra ${ }^{3}$ \& Samuel Aparicio ${ }^{1,2,5}$
}

\begin{abstract}
Recent advances in next generation sequencing ${ }^{1-4}$ have made it possible to precisely characterize all somatic coding mutations that occur during the development and progression of individual cancers. Here we used these approaches to sequence the genomes $(>43$ fold coverage) and transcriptomes of an oestrogen-receptor- $\alpha$ positive metastatic lobular breast cancer at depth. We found 32 somatic non-synonymous coding mutations present in the metastasis, and measured the frequency of these somatic mutations in DNA from the primary tumour of the same patient, which arose 9 years earlier. Five of the 32 mutations (in ABCB11, HAUS3, SLC24A4, SNX4 and PALB2) were prevalent in the DNA of the primary tumour removed at diagnosis 9 years earlier, six (in KIF1C, USP28, MYH8, MORC1, KIAA1468 and RNASEH2A) were present at lower frequencies $(1-13 \%), 19$ were not detected in the primary tumour, and two were undetermined. The combined analysis of genome and transcriptome data revealed two new RNAediting events that recode the amino acid sequence of SRP9 and COG3. Taken together, our data show that single nucleotide mutational heterogeneity can be a property of low or intermediate grade primary breast cancers and that significant evolution can occur with disease progression.
\end{abstract}

Lobular breast cancer is an oestrogen-receptor-positive $\left(\mathrm{ER}^{+}\right.$, also known as ESR $1^{+}$) subtype of breast cancer (approximately $15 \%$ of all breast cancers). It is usually of low-intermediate histological grade and can recur many years after initial diagnosis. To interrogate the genomic landscape of this class of tumour, we re-sequenced ${ }^{1-4}$ the DNA from a metastatic lobular breast cancer specimen (89\% tumour cellularity; Supplementary Fig. 1) at approximately 43.1-fold aligned, haploid reference genome coverage (120.7 gigabases (Gb) aligned paired-end sequence; Supplementary Fig. 2, Table 1 and Supplementary Methods). Deep high-throughput transcriptome sequencing $(\text { RNA-seq })^{5}$ performed on the same sample generated 160.9-million reads that could be aligned (Supplementary Table 1, see also Supplementary Fig. 2 and Supplementary Methods). The saturation of the genome (Table 1) and RNA-seq (Supplementary Table 1) libraries for single nucleotide variant (SNV) detection is discussed in Supplementary Information. The aligned (hg18) reads were used to identify (Supplementary Fig. 2) the presence of genomic aberrations, including SNVs (Supplementary Table 2), insertions/deletions (indels), gene fusions, translocations, inversions and copy number alterations (Supplementary Methods). We examined predicted coding indels and predicted inversions (coding or non-coding; Supplementary Methods); however, all of the events that were validated by Sanger re-sequencing were also present in the germ line (Supplementary Tables 3 and 4). None of the 12 predicted gene fusions revalidated. We also computed the segmental copy number (Supplementary Methods and Supplementary Table 5a) from aligned reads, and revalidated high level amplicons by fluorescence in situ hybridization (FISH) (Supplementary Table 5b), revealing the presence of a new low-level amplicon in the INSR locus (Supplementary Fig. 3).

We identified coding SNVs from aligned reads, using a Binomial mixture model, SNVMix (Supplementary Table 2, Methods and Supplementary Appendix 1). From the RNA-seq (WTSS-PE) and genome (WGSS-PE) libraries we predicted 1,456 new coding nonsynonymous SNVMix variants (Supplementary Table 2). After the removal of pseudogene and HLA sequences ( 1,178 positions remaining) and after primer design, we re-sequenced (Sanger amplicons) 1,120 non-synonymous coding SNV positions in the tumour DNA and normal lymphocyte DNA. Some 437 positions (268 unique to WGSS-PE, 15 unique to WTSS-PE, and 154 in common) were confirmed as non-synonymous coding variants. Of these, 405 were new

Table 1 | Summary of sequence library coverage

\begin{tabular}{lll}
\hline & WGSS-PE & WTSS-PE \\
\hline Total number of reads & $2,922,713,774$ & $182,532,650$ \\
Total nucleotides (Gb) & 140.991 & 7.108 \\
Number of aligned reads & $2,502,465,226$ & $160,919,484$ \\
Aligned nucleotides (Gb) & 120.718 & 6.266 \\
Estimated error rate & 0.021 & 0.013 \\
Estimated depth (non-gap & 43.114 & NA \\
regions) & & \\
Canonically aligned reads & $2,294,067,534$ & $109,093,616$ \\
Exons covered & 93.5 at $>10$ reads; & 82,200 at 10 reads (see also \\
& 95.7 at $>5$ reads & Supplementary Table 1) \\
Reads aligned canonically (\%) & 78.49 & 67.79 \\
Unaligned reads & $420,248,548$ & $21,613,166$ \\
Mean read length (bp) & 48.24 & 38.94 \\
\hline
\end{tabular}

The WGSS-PE column shows the genome paired-end read coverage for DNA from the metastatic pleural effusion sample. The WTSS-PE column shows coverage for the

complementary DNA reads from the matched transcriptome libraries of the metastatic pleural effusion. Coverage of exon bases in the reference genome (hg18) is shown at 5 or more reads per position, and 10 or more reads per position for the metastatic genome. bp, base pairs; NA, not applicable.

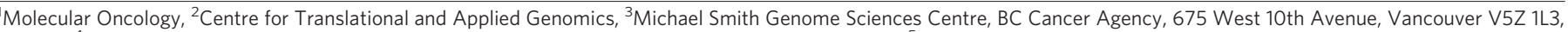
Canada. ${ }^{4}$ Medical Oncology, BC Cancer Agency, 600 West 10th Avenue, Vancouver V5Z 1L3, Canada. ${ }^{5}$ Department of Pathology, University of British Columbia, G227-2211

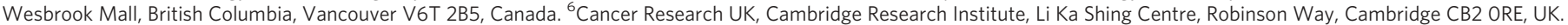
${ }^{7}$ Deeley Research Centre, BC Cancer Agency, Victoria V8R 6V5, Canada.

*These authors contributed equally to this work.
} 
Table 2 | Somatic coding sequence SNVs validated by Sanger sequencing

\begin{tabular}{|c|c|c|c|c|c|c|c|c|c|}
\hline Gene & Description & Position & Source & $\begin{array}{l}\text { Allele } \\
\text { change }\end{array}$ & $\begin{array}{l}\text { Amino } \\
\text { acid } \\
\text { change }\end{array}$ & $\begin{array}{l}\text { Protein domain } \\
\text { affected }\end{array}$ & $\begin{array}{l}\text { Expression } \\
\text { (sequenced } \\
\text { bases per } \\
\text { exonic base) }\end{array}$ & $\begin{array}{l}\text { Allelic } \\
\text { expression } \\
\text { bias ( } R, N R \\
\text { allele) }\end{array}$ & $\begin{array}{l}\text { Copy number } \\
\text { classification } \\
\text { (HMM state) }\end{array}$ \\
\hline$A B C B 11$ & $\begin{array}{l}\text { Bile salt export pump } \\
\text { (ATP-binding cassette } \\
\text { sub-family B member 11) }\end{array}$ & 2:169497197 & WGSS & $C>T$ & $\mathrm{R}>\mathrm{H}$ & $\begin{array}{l}\text { Transmembrane } \\
\text { helix } 3\end{array}$ & 0.3 & 1,1 & $\begin{array}{l}\text { Amplification } \\
\text { (4) }\end{array}$ \\
\hline HAUS3 & $\begin{array}{l}\text { HAUS3 coiled-coil } \\
\text { protein (C4orf15) }\end{array}$ & 4:2203607 & WGSS, WTSS & $C>T$ & $V>M$ & Unknown & 14.1 & 4,23 & Neutral (2) \\
\hline CDC6 & $\begin{array}{l}\text { Cell division control } \\
\text { protein } 6 \text { homologue }\end{array}$ & 17:35701114 & WGSS, WTSS & $G>A$ & $E>K$ & $\begin{array}{l}\text { N-terminal, } \\
\text { unknown }\end{array}$ & 2.7 & 3,3 & $\begin{array}{l}\text { Amplification } \\
\text { (4) }\end{array}$ \\
\hline CHD3 & $\begin{array}{l}\text { Chromodomain- } \\
\text { helicase-DNA-binding } \\
\text { protein } 3\end{array}$ & 17:7751231 & WGSS & $G>A$ & $E>K$ & $\begin{array}{l}\text { Unknown, } \\
\text { C-terminal }\end{array}$ & 3.9 & $\begin{array}{l}41,11 \\
(Q<0.01)\end{array}$ & Neutral (2) \\
\hline DLG4 & $\begin{array}{l}\text { Disks large } \\
\text { homologue } 4\end{array}$ & 17:7052251 & WGSS & $G>A$ & $P>L$ & $\begin{array}{l}\text { Unknown, } \\
\text { N-terminal }\end{array}$ & 5.5 & 7,1 & Neutral (2) \\
\hline ERBB2 & $\begin{array}{l}\text { Receptor tyrosine- } \\
\text { protein kinase erb-b2 }\end{array}$ & 17:35133783 & WGSS, WTSS & $C>G$ & $I>M$ & Kinase domain & 67.1 & 62,35 & $\begin{array}{l}\text { Amplification } \\
\text { (4) }\end{array}$ \\
\hline FGA & Fibrinogen alpha chain & $4: 155726802$ & WGSS & $C>T$ & $W>$ stop & $\begin{array}{l}\text { Fibrinogen } a / b / c \\
\text { domain }\end{array}$ & 0.01 & NA & Gain (3) \\
\hline GOLGA4* & $\begin{array}{l}\text { Golgin subfamily } \\
\text { A member } 4\end{array}$ & 3:37267947 & WGSS, WTSS & $G>C$ & $E>Q$ & $\begin{array}{l}\text { Unknown, } \\
\text { N-terminal }\end{array}$ & 111.8 & 37,12 & Gain (3) \\
\hline GSTCD & $\begin{array}{l}\text { Glutathione S-transferase } \\
\text { C-terminal domain- } \\
\text { containing protein }\end{array}$ & 4:106982671 & WGSS, WTSS & $G>C$ & $E>Q$ & $\begin{array}{l}\text { Unknown, } \\
\text { C-terminal }\end{array}$ & 23.2 & 23,8 & Neutral (2) \\
\hline KIAA1468* & $\begin{array}{l}\text { LisH domain and HEAT } \\
\text { repeat-containing protein }\end{array}$ & $18: 58076768$ & WGSS, WTSS & $G>C$ & $R>T$ & ARM type fold & 36.1 & 23,11 & Neutral (2) \\
\hline KIF1C & $\begin{array}{l}\text { Kinesin-like protein } \\
\text { KIF1C }\end{array}$ & $17: 4848025$ & WGSS, WTSS & $G>C$ & $K>N$ & $\begin{array}{l}\text { Kinesin motor } \\
\text { domain }\end{array}$ & 28.5 & 16,13 & Neutral (2) \\
\hline KLHL4 & Kelch-like protein 4 & X:86659878 & WGSS & $C>T$ & $S>L$ & $\begin{array}{l}\text { Unknown, } \\
\text { N-terminal }\end{array}$ & 1.7 & 1,0 & Neutral (2) \\
\hline MYH8 & $\begin{array}{l}\text { Myosin } 8 \text { (myosin heavy } \\
\text { chain } 8 \text { ) }\end{array}$ & $17: 10248420$ & WGSS & $C>G$ & $M>1$ & $\begin{array}{l}\text { Actin-interacting } \\
\text { protein domain }\end{array}$ & 0 & NA & Neutral (2) \\
\hline PALB2 & $\begin{array}{l}\text { Partner and localizer } \\
\text { of BRCA2 }\end{array}$ & $16: 23559936$ & WGSS & $T>G$ & $E>A$ & $\begin{array}{l}\mathrm{N} \text {-terminal } \\
\text { prefolding }\end{array}$ & 13.0 & NA & $\begin{array}{l}\text { Amplification } \\
\text { (4) }\end{array}$ \\
\hline PKDREJ & $\begin{array}{l}\text { Polycystic kidney } \\
\text { disease and receptor for } \\
\text { egg-jelly-related protein }\end{array}$ & $22: 45035285$ & WGSS & $C>G$ & $E>Q$ & Unknown & 0.1 & NA & Gain (3) \\
\hline RASEF & $\begin{array}{l}\text { RAS and EF-hand } \\
\text { domain-containing } \\
\text { protein }\end{array}$ & 9:84867250 & WTSS & $G>A$ & $S>L$ & $\begin{array}{l}\text { EF-hand } \mathrm{Ca}^{2+} \\
\text { binding motif }\end{array}$ & 65.0 & 3,2 & Gain (3) \\
\hline RNASEH2A & $\begin{array}{l}\text { Ribonuclease } \mathrm{H} 2 \\
\text { subunit } \mathrm{A}(\mathrm{EC} \text { 3.1.26.4) }\end{array}$ & 19:12785252 & WGSS, WTSS & $G>A$ & $\mathrm{R}>\mathrm{H}$ & $\begin{array}{l}\text { Unknown, } \\
\text { C-terminal }\end{array}$ & 5.3 & 2,2 & Neutral (2) \\
\hline RNF220 & $\begin{array}{l}\text { RING finger protein } \\
\text { C1orf164 }\end{array}$ & 1:44650831 & WGSS & $G>A$ & $\mathrm{D}>\mathrm{N}$ & $\begin{array}{l}\text { Unknown, } \\
\text { N-terminal }\end{array}$ & 16.1 & NA & Neutral (2) \\
\hline SP1 & Transcription factor Sp1 & $12: 52063157$ & WGSS & $G>C$ & $E>Q$ & $\begin{array}{l}\text { Glu-rich } \\
\text { N-terminal domain }\end{array}$ & 57.3 & $\begin{array}{l}40,10 \\
(Q<0.01)\end{array}$ & $\begin{array}{l}\text { Amplification } \\
\text { (4) }\end{array}$ \\
\hline USP28 & $\begin{array}{l}\text { Ubiquitin carboxyl- } \\
\text { terminal hydrolase } 28\end{array}$ & 11:113185109 & WGSS, WTSS & $C>T$ & $\mathrm{D}>\mathrm{N}$ & Unknown & 12.5 & 3,7 & Gain (3) \\
\hline C11orf10 & $\begin{array}{l}\text { UPF0197 } \\
\text { transmembrane } \\
\text { protein C11orf10 }\end{array}$ & $11: 61313958$ & WGSS & $G>A$ & $\mathrm{~T}>\mathrm{I}$ & $\begin{array}{l}\text { Transmembrane } \\
\text { domain }\end{array}$ & 28.9 & 13,3 & $\begin{array}{l}\text { Amplification } \\
\text { (4) }\end{array}$ \\
\hline THRSP & $\begin{array}{l}\text { Thyroid hormone- } \\
\text { inducible hepatic protein }\end{array}$ & $11: 77452594$ & WGSS & $C>T$ & $\mathrm{R}>\mathrm{C}$ & Unknown & 0.3 & NA & Gain (3) \\
\hline SCEL & Sciellin & 13:77076497 & WGSS & $A>G$ & $K>R$ & Unknown & 0.3 & 1,0 & Gain (3) \\
\hline SLC24A4 & $\begin{array}{l}\mathrm{Na}^{+} / \mathrm{K}^{+} / \mathrm{Ca}^{2+}- \\
\text { exchange protein } 4\end{array}$ & $14: 92018836$ & WGSS & $G>A$ & $V>1$ & $\begin{array}{l}\text { Transmembrane } \\
\text { domain }\end{array}$ & 1.2 & 1,0 & $\begin{array}{l}\text { Amplification } \\
(4)\end{array}$ \\
\hline COLIA1 & $\begin{array}{l}\text { Collagen alpha-1(I) } \\
\text { chain precursor }\end{array}$ & $17: 45625043$ & WGSS & $C>T$ & $G>D$ & Pro-rich domain & 80.0 & $\begin{array}{l}24,0 \\
(Q<0.01)\end{array}$ & $\begin{array}{l}\text { Amplification } \\
\text { (4) }\end{array}$ \\
\hline KIAA1772 & GREB1-like protein & 18:17278222 & WGSS & $A>G$ & $D>G$ & Unknown & 2.8 & 4,1 & Neutral (2) \\
\hline CCDC117 & $\begin{array}{l}\text { Coiled-coil domain- } \\
\text { containing protein } 117\end{array}$ & 22:27506951 & WGSS & $\mathrm{G}>\mathrm{C}$ & $K>N$ & Unknown & 12.9 & 2,0 & Neutral (2) \\
\hline $\begin{array}{l}\text { RP1- } \\
32110.10\end{array}$ & Novel protein & $22: 43140252$ & WGSS & $G>C$ & $E>Q$ & Unknown & 0 & NA & Gain (3) \\
\hline MORC1 & $\begin{array}{l}\text { MORC family CW-type } \\
\text { zinc finger protein } 1\end{array}$ & 3:110271286 & WGSS & $G>A$ & $A>V$ & Coiled-coil & 0.1 & NA & Gain (3) \\
\hline SNX4 & Sorting nexin 4 & $3: 126721688$ & WGSS & $C>T$ & $\mathrm{D}>\mathrm{N}$ & $\begin{array}{l}\text { Unknown, } \\
\text { N-terminal }\end{array}$ & 43.4 & NA & Gain (3) \\
\hline LEPREL1 & $\begin{array}{l}\text { Prolyl 3-hydroxylase } 2 \\
\text { precursor (EC 1.14.11.7) }\end{array}$ & 3:191172415 & WGSS & $\mathrm{T}>\mathrm{C}$ & $E>G$ & $\begin{array}{l}\text { Hydroxylase } \\
\text { domain }\end{array}$ & 1.1 & NA & Gain (3) \\
\hline WDR59* & $\begin{array}{l}\text { WD repeat-containing } \\
\text { protein } 59\end{array}$ & $16: 73500342$ & WTSS & $C>T$ & $M>1$ & $\begin{array}{l}\text { Unknown, } \\
\text { C-terminal }\end{array}$ & 17.3 & 6,5 & Neutral (2) \\
\hline
\end{tabular}

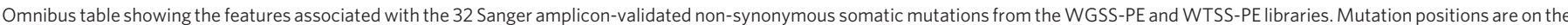
basis of reference genome $\mathrm{hg} 18$. The nucleotide substitutions are shown as reference $>$ variant. The amino acid change is shown as reference $>$ variant amino acid. If the mutation occurs in a

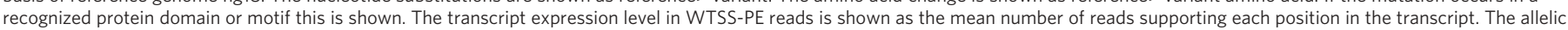

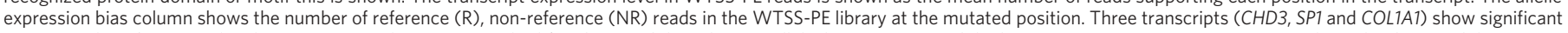
expression bias (annotated with $Q<0.01$, Supplementary Methods) in favour of the reference allele; however, none of the heterozygous somatic mutations were biased in favour of the non-

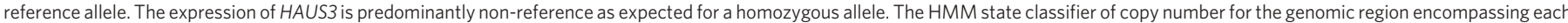
mutation position is shown in the last column, as state (state number). C-terminal, carboxy-terminal; N-terminal, amino-terminal.

*Genes showing alternative splicing. 
germline alleles and 32 were revealed as non-synonymous coding somatic point mutations (Table 2). Of the 32 somatic mutations, 30 were present in WGSS-PE and/or WTSS-PE, whereas two were detected from the WTSS library sequence alone (Table 2). None of the 32 genes were found in common with the CAN breast genes ${ }^{6}$, which were discovered from $\mathrm{ER}^{-}$cell lines. Eleven genes appear in the current release of $\mathrm{COSMIC}^{7}$ (CHD3, SP1, PALB2, ERBB2, USP28, KLHL4, CDC6, KIAA1468, RNF220, COL1A1 and SNX4) but with mutations at different positions. We examined the population frequency of the somatic mutation positions for PALB2, ERBB2, USP28, CDC6, CHD3, HAUS3 (previously known as C4orf15), SP1, KIAA1468 and DLG4 in a further 192 breast cancers (Supplementary Methods; 112 lobular, 80 ductal). None of these 192 breast cancers showed identical mutations to those described here; however, 3 out of 192 cases ( 2 lobular, 1 ductal) contained neighbouring nonsynonymous variants/deletions affecting the ERBB2 kinase domain (Supplementary Fig. 4). Interestingly, 2 out of 192 cases (both lobular) contained two different heterozygous truncating variants in HAUS3: chr4:2203685 G>T on minus strand, GAG $>$ TAG (Glu>stop), and chr4:2203483 C $>$ G on minus strand, TCA $>$ TGA (Ser $>$ stop) (Supplementary Fig. 5). Notably, HAUS3 is a member of the recently described ${ }^{8-10}$ multiprotein augmin complex, the function of which is required for genome stability mediated by appropriate kinetochore attachment and centrosome morphogenesis.

To determine how many of the somatic non-synonymous coding sequence mutations were already present at diagnosis 9 years earlier, we next examined genomic DNA from the primary tumour directly, by a single molecule frequency counting experiment (Supplementary Methods $)^{4}$. Twenty-eight of the 32 mutations yielded amplicons compatible with Illumina sequencing (Supplementary Methods), and two extra mutations were sampled by Sanger sequencing
(Supplementary Fig. 5). As controls we selected 36 heterozygous germline SNVs at random. The PCR amplicons for known germline and somatic mutations were sequenced on an Illumina device. After alignment, the observed counts of reference and non-reference bases at the target position were compared using the Binomial exact test. To calibrate the expected mean of the Binomial distribution, we used the non-reference allele frequency from positions -5 to +5 surrounding (but not including) the target position (Supplementary Table $6 \mathrm{a}, \mathrm{b}$ ), where only reference bases should be called. Unequal segmental amplification/deletion in the genome may contribute to a departure from the theoretical ratio of 0.5 for a heterozygous allele. As a result, amplicons from heterozygous germline alleles showed occasional measured frequencies of between 0.2 and 0.8 in both the primary and metastatic tumour DNA (Table 3 and Supplementary Table 7 ), but with a modal frequency around 0.5 , as expected. In the metastatic genomic DNA the somatic mutations showed frequencies of between 0.2 and 0.79 (Table 3). Notably, the somatic coding mutation positions examined in the primary tumour showed three patterns of abundance: prevalent, rare and undetectable (Table 3). Mutations in ABCB11, PALB2 and SLC24A4 were detected at prevalent frequencies for heterozygous mutations $(\geq 0.2$, the lowest value seen for known germline alleles) given a $73 \%$ tumour content. The frequency of the mutation in HAUS3 was 0.79 , consistent with it being a prevalent homozygous mutation, also confirmed by Sanger sequencing (Supplementary Fig. 5). Sanger amplicon sequencing showed that the SNX4 somatic mutation was also present in the primary tumour, whereas the KIAA1772 (also known as GREB1L) mutation was not. Six mutations (KIF1C, USP28, MORC1, MYH8, KIAA1468 and RNASEH2A) showed statistically significant $(P<0.01$, Binomial exact test $)$ intermediate frequencies of between $1 \%$ and $13 \%$ (Table 3), suggesting that these mutations were

Table 3 | Frequency of germline and somatic alleles in the metastatic and primary genomes

\begin{tabular}{|c|c|c|c|c|c|c|c|c|c|c|c|}
\hline Position & Locus & $\mathrm{R}$ & NR & $\begin{array}{l}\text { Primary } \\
\text { depth }\end{array}$ & $\begin{array}{l}\text { Primary } \\
\text { NR ratio }\end{array}$ & $\begin{array}{l}\text { Primary } \\
P \text { value }\end{array}$ & Primary status & $\begin{array}{l}\text { Metastasis } \\
\text { depth }\end{array}$ & $\begin{array}{l}\text { Metastasis } \\
\text { NR ratio }\end{array}$ & M & $\begin{array}{l}\text { Copy number } \\
\text { classification (HMM state) }\end{array}$ \\
\hline 4:2203607 & HAUS3 & C & $\mathrm{T}$ & 5700 & 0.5472 & 0.0000 & Dominant & 762 & 0.7874 & $\mathrm{~S}$ & Neutral (2) \\
\hline $16: 23559936$ & PALB2 & $\mathrm{T}$ & G & 115 & 0.4957 & 0.0000 & Dominant & 669 & 0.4350 & $\mathrm{~S}$ & Amplification (4) \\
\hline 2:169497197 & $A B C B 11$ & C & $\mathrm{T}$ & 506 & 0.3261 & 0.0000 & Dominant & 959 & 0.3691 & $\mathrm{~S}$ & Amplification (4) \\
\hline 14:92018836 & SLC24A4 & G & $A$ & 13347 & 0.2341 & 0.0000 & Dominant & 13670 & 0.3518 & $\mathrm{~S}$ & Amplification (4) \\
\hline 17:10248420 & MYH8 & $\mathrm{C}$ & G & 10657 & 0.1353 & 0.0000 & Subdominant & 1797 & 0.5932 & $\mathrm{~S}$ & Neutral (2) \\
\hline 3:110271286 & MORC1 & G & $A$ & 24572 & 0.0468 & 0.0000 & Subdominant & 32273 & 0.4107 & $\mathrm{~S}$ & Gain (3) \\
\hline $17: 4848025$ & KIF1C & G & $A$ & 8587 & 0.0107 & 0.0000 & Subdominant & 2272 & 0.3077 & $\mathrm{~S}$ & Neutral (2) \\
\hline 11:113185109 & USP28 & $\mathrm{C}$ & $\mathrm{T}$ & 6654 & 0.0095 & 0.0000 & Subdominant & 1387 & 0.4484 & $\mathrm{~S}$ & Gain (3) \\
\hline $18: 58076768$ & KIAA1468 & G & $A$ & 719 & 0.0083 & 0.0020 & Subdominant & 1056 & 0.3059 & $\mathrm{~S}$ & Neutral (2) \\
\hline $19: 12785252$ & RNASEH2A & G & $A$ & 6537 & 0.0029 & 0.0276 & Subdominant & 1497 & 0.2806 & $\mathrm{~S}$ & Neutral (2) \\
\hline 4:106982671 & GSTCD & G & $\mathrm{T}$ & 7273 & 0.0008 & 0.9885 & Absent & 2208 & 0.2174 & $\mathrm{~S}$ & Neutral (2) \\
\hline $17: 35701114$ & CDC6 & G & $\mathrm{T}$ & 4894 & 0.0008 & 0.9733 & Absent & 4208 & 0.3577 & $\mathrm{~S}$ & Amplification (4) \\
\hline $17: 7751231$ & $\mathrm{CHD} 3$ & G & $A$ & 9665 & 0.0007 & 0.9981 & Absent & 1737 & 0.2671 & $\mathrm{~S}$ & Neutral (2) \\
\hline 4:155726802 & $F G A$ & C & $\mathrm{T}$ & 5756 & 0.0007 & 0.9911 & Absent & 2287 & 0.2755 & $\mathrm{~S}$ & Gain (3) \\
\hline 17:7052251 & DLG4 & G & $A$ & 4383 & 0.0007 & 0.9835 & Absent & 706 & 0.3272 & $\mathrm{~S}$ & Neutral (2) \\
\hline $3: 37267947$ & GOLGA4 & G & $\mathrm{T}$ & 13051 & 0.0006 & 0.9999 & Absent & 3262 & 0.2235 & $\mathrm{~S}$ & Gain (3) \\
\hline $9: 84867250$ & RASEF & G & $\mathrm{T}$ & 1690 & 0.0006 & 0.9500 & Absent & 796 & 0.3656 & $\mathrm{~S}$ & Gain (3) \\
\hline $17: 35133783$ & ERBB2 & C & $A$ & 3736 & 0.0005 & 0.9899 & Absent & 1722 & 0.3612 & $\mathrm{~S}$ & Amplification (4) \\
\hline$X: 86659878$ & KLHL4 & C & $\mathrm{T}$ & 6561 & 0.0005 & 0.9993 & Absent & 977 & 0.3153 & $\mathrm{~S}$ & Neutral (2) \\
\hline 3:191172415 & LPREL1 & $\mathrm{T}$ & C & 11963 & 0.0004 & 1.0000 & Absent & 8381 & 0.2148 & $\mathrm{~S}$ & Gain (3) \\
\hline $16: 73500342$ & WDR59 & C & $\mathrm{T}$ & 4846 & 0.0004 & 0.9982 & Absent & 1396 & 0.2629 & $\mathrm{~S}$ & Neutral (2) \\
\hline $1: 44650831$ & RNF220 & G & $A$ & 8160 & 0.0004 & 0.9999 & Absent & 967 & 0.2203 & $\mathrm{~S}$ & Neutral (2) \\
\hline $22: 45035285$ & PKDREJ & $\mathrm{C}$ & $\mathrm{T}$ & 6674 & 0.0003 & 0.9999 & Absent & 1230 & 0.3366 & $\mathrm{~S}$ & Gain (3) \\
\hline 11:61313958 & C11ORF10 & G & $A$ & 116705 & 0.0003 & 1.0000 & Absent & 14354 & 0.4651 & $\mathrm{~S}$ & Amplification (4) \\
\hline $12: 52063157$ & SP1 & G & $\mathrm{T}$ & 7732 & 0.0003 & 1.0000 & Absent & 2011 & 0.2193 & $\mathrm{~S}$ & Amplification (4) \\
\hline $11: 77452594$ & THRSP & $\mathrm{C}$ & $\mathrm{T}$ & 24219 & 0.0002 & 1.0000 & Absent & 40652 & 0.4750 & $\mathrm{~S}$ & Gain (3) \\
\hline $17: 45625043$ & COLIA1 & C & $A$ & 26343 & 0.0001 & 1.0000 & Absent & 32259 & 0.2543 & $\mathrm{~S}$ & Amplification (4) \\
\hline 13:77076497 & SCEL & A & G & 49 & 0.0000 & 1.0000 & Absent & 187 & 0.5722 & $S$ & Gain (3) \\
\hline $19: 9314428$ & - & A & G & 176 & 0.5057 & 0.0000 & Present & 321 & 0.4953 & G & Neutral (2) \\
\hline $4: 130144460$ & - & $A$ & $\mathrm{~T}$ & 2020 & 0.2188 & 0.0000 & Present & 2081 & 0.3099 & G & Neutral (2) \\
\hline 8:27835012 & - & G & $A$ & 13587 & 0.8602 & 0.0000 & Present & 10781 & 0.6667 & G & Deletion (1) \\
\hline $6: 32908543$ & - & $\mathrm{C}$ & $\mathrm{T}$ & 4718 & 0.7484 & 0.0000 & Present & 16370 & 0.4897 & G & Amplification (4) \\
\hline $20: 43363061$ & - & G & $A$ & 5950 & 0.5249 & 0.0000 & Present & 5540 & 0.5049 & G & Amplification (4) \\
\hline 4:8672089 & - & G & $A$ & 381 & 1.0000 & 0.0000 & Present & 2850 & 0.8032 & G & Gain (3) \\
\hline $16: 1331138$ & - & C & $\mathrm{T}$ & 677 & 0.4963 & 0.0000 & Present & 554 & 0.6245 & G & High-level amplicon (5) \\
\hline
\end{tabular}

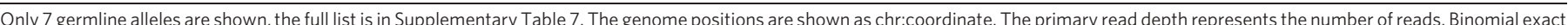

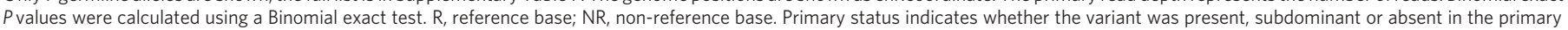
tumour. Column M denotes somatic (S) or germline $(\mathrm{G})$ single nucleotide variants in the metastasis. HMM state refers to the metastasis. 


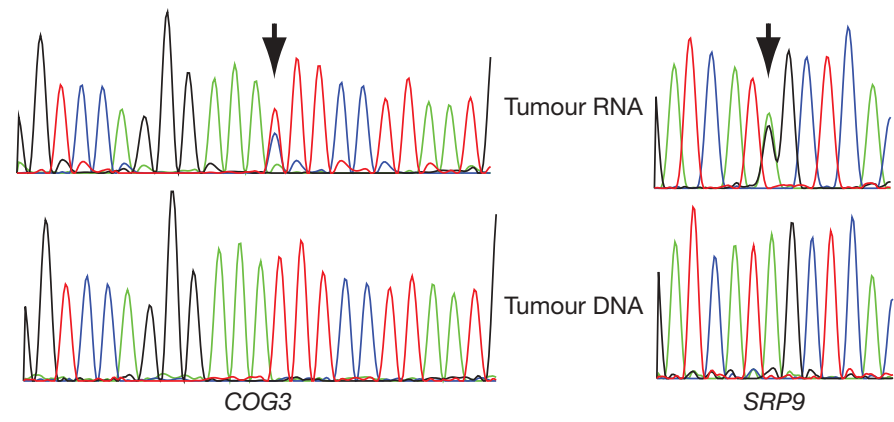

Figure 1 | RNA editing in COG3 and SRP9. Sanger sequence traces from the non-synonymous editing positions in COG3 and SRP9. The editing position is arrowed. Top trace is tumour RNA, bottom trace tumour DNA. The editing positions were confirmed with reverse strand reads (not shown).

restricted to minor subclones of tumour cells. The remaining 19 out of 30 of the somatic coding mutations were not detected in the primary tumour DNA. Thus, significant heterogeneity in tumour somatic mutation content existed in the primary tumour at diagnosis. In contrast with the recently reported sequence of cytogenetically normal acute myeloid leukaemia (AML) tumour ${ }^{4}$, significant evolution of coding mutational content occurred between primary and metastasis. It is unknown whether the 19 mutations present in the metastasis, but not detected in the primary, were a consequence of radiation therapy or innate tumour progression.

We also examined how the transfer of information from the nuclear genome to proteins was modified by alternative splicing (Supplementary Table 8 and Supplementary Fig. 6), biased allelic expression (Supplementary Table 9) and RNA editing. At the single nucleotide level, RNA-editing enzymes (which can be regulated by oestrogens ${ }^{11}$ ) may also recode transcripts resulting in a proteome divergent from the genome ${ }^{12-15}$. Interestingly, the ADAR enzymeone of the principal RNA-editing enzymes that mediates $A \rightarrow I(G)$ edits-was one of the top 5\% of genes expressed (145.6 reads per base, Supplementary Table 10), and the only editing enzyme expressed at a high level. We searched for potential editing events (Methods) and found 3,122 candidate edits in 1,637 gene loci (Supplementary Table 11). Some 526 out of 3,122 candidate edits are non-synonymous changes and 232 are synonymous changes (with the remainder affecting untranslated regions). We revalidated independently (Supplementary Methods) by Sanger sequencing 75 editing events in 12 gene loci from the lobular metastasis (Supplementary Table 12 and see trace data at http:// molonc.bccrc.ca/). Two genes, COG3 and SRP9 (Fig. 1), showed confirmed high frequency non-synonymous transcript editing, resulting in variant protein sequences. These observations emphasize the importance of integrating RNA-seq data with tumour genomes in assessing protein variation.

The coding mutation landscape of breast cancers has, so far, been mostly determined from $\mathrm{ER}^{-}$metastatic cell lines/samples, ${ }^{6,16}$, and has suggested the presence of large numbers of passenger events as well as drivers. Our results show the importance of sequencing samples of tumour cell populations early as well as late in the evolution of tumours, and of estimating allele frequency in tumour genomes. Our observations suggest that the sequencing of primary breast cancers and pre-invasive malignancy may reveal significantly fewer candidates for tumour initiating mutations.

\section{METHODS SUMMARY}

Paired-end reads were assigned quality scores and aligned to the reference genome (hg18) using Maq ${ }^{17}$ (Supplementary Methods and Supplementary Fig. 2). For identification of SNVs we used a simple Binomial mixture model, SNVMix (Supplementary Appendix 1), which assigns a probability to each base position as homozygous reference (aa), heterozygous non-reference (ab) and homozygous non-reference (bb), based on the occurrence of reference (hg18) and non-reference bases at each aligned position. This model was calibrated initially, using high confidence allele calls from Affymetrix SNP6.0 hybridization of tumour and normal DNA. We estimated the receiver operating characteristic (ROC) performance (Supplementary Fig. 8) and determined that an SNVMix threshold of $P=0.77$ for $(\mathrm{ab})$ or ( $\mathrm{bb}$ ) for a non-reference call would yield a false discovery rate (FDR) of $1 \%$. For the RNA-seq library, a threshold of $P=0.53$ was used (Supplementary Fig. 8; FDR $=0.01$ ) to call non-reference positions. Nonreference positions were then filtered for known variants against the sources of germline variation, the single nucleotide polymorphism database (dbSNP) and the completed individual genomes ${ }^{18,19}$ (Supplementary Table 2). Saturation of the libraries for SNV discovery was determined by random re-sampling (Supplementary Fig. 9 and Supplementary Methods). Segmental copy number was inferred with a hidden Markov model (HMM) method (Supplementary Table 4a, b and Supplementary Methods).

We searched for RNA-editing events by examining all very high confidence $(P(\mathrm{ab})+P(\mathrm{bb})>0.9)$ SNVMix predictions from the RNA-seq library of the metastatic tumour, that were not found with extreme confidence $(P(\mathrm{aa})>0.99$, derived from the SNVMix receiver operating curve at FDR $=0.01$ ) at the same positions in the metastatic tumour genome library.

\section{Received 4 September; accepted 10 September 2009.}

1. Campbell, P. J. et al. Identification of somatically acquired rearrangements in cancer using genome-wide massively parallel paired-end sequencing. Nature Genet. 40, 722-729 (2008).

2. Bentley, D. R. et al. Accurate whole human genome sequencing using reversible terminator chemistry. Nature 456, 53-59 (2008).

3. Morin, R. et al. Profiling the HeLa S3 transcriptome using randomly primed cDNA and massively parallel short-read sequencing. Biotechniques 45, 81-94 (2008).

4. Ley, T. J. et al. DNA sequencing of a cytogenetically normal acute myeloid leukaemia genome. Nature 456, 66-72 (2008).

5. Mortazavi, A., Williams, B. A., McCue, K., Schaeffer, L. \& Wold, B. Mapping and quantifying mammalian transcripomes by RNA-Seq. Nature Methods 5, 621-628 (2008).

6. Wood, L. D. et al. The genomic landscapes of human breast and colorectal cancers. Science 318, 1108-1113 (2007).

7. Forbes, S. A. et al. The Catalogue of Somatic Mutations in Cancer (COSMIC). Curr. Protoc. Hum. Genet. Unit 10.11, doi:10.1002/0471142905.hg1011s57 (2008).

8. Goshima, G., Mayer, M., Zhang, N., Stuurman, N. \& Vale, R. D. Augmin: a protein complex required for centrosome-independent microtubule generation within the spindle. J. Cell Biol. 181, 421-429 (2008).

9. Meireles, A. M., Fisher, K. H., Colombie, N., Wakefield, J. G. \& Ohkura, H. Wac: a new Augmin subunit required for chromosome alignment but not for acentrosomal microtubule assembly in female meiosis. J. Cell Biol. 184, 777-784 (2009).

10. Lawo, S. et al. HAUS, the 8-subunit human Augmin complex, regulates centrosome and spindle integrity. Curr. Biol. 19, 816-826 (2009).

11. Pauklin, S., Sernandez, I. V., Bachmann, G., Ramiro, A. R. \& Petersen-Mahrt, S. K. Estrogen directly activates AID transcription and function. J. Exp. Med. 206, 99-111 (2009).

12. Blow, M., Futreal, P. A., Wooster, R. \& Stratton, M. R. A survey of RNA editing in human brain. Genome Res. 14, 2379-2387 (2004)

13. Athanasiadis, A., Rich, A. \& Maas, S. Widespread A-to-I RNA editing of Alucontaining mRNAs in the human transcriptome. PLoS Biol. 2, e391 (2004).

14. Maas, S., Kawahara, Y., Tamburro, K. M. \& Nishikura, K. A-to-I RNA editing and human disease. RNA Biol. 3, 1-9 (2006)

15. Li, J. B. et al. Genome-wide identification of human RNA editing sites by parallel DNA capturing and sequencing. Science 324, 1210-1213 (2009).

16. Greenman, C. et al. Patterns of somatic mutation in human cancer genomes. Nature 446, 153-158 (2007).

17. Li, H., Ruan, J. \& Durbin, R. Mapping short DNA sequencing reads and calling variants using mapping quality scores. Genome Res. 18, 1851-1858 (2008).

18. Wang, J. et al. The diploid genome sequence of an Asian individual. Nature 456, 60-65 (2008).

19. Wheeler, D. A. et al. The complete genome of an individual by massively parallel DNA sequencing. Nature 452, 872-876 (2008).

Supplementary Information is linked to the online version of the paper at www.nature.com/nature.

Acknowledgements We thank C. Eaves and M. Pollak for comments on earlier versions of the manuscript. We thank and acknowledge the patients of the BC Cancer Agency for donations of tumour tissues to the TTR-BREAST tumour banking program. S.A. is supported by a Canada Research Chair in Molecular Oncology, S.P.S., J.K., L.P., A.B. and T.P. are supported by Michael Smith Foundation for Health Research awards. R.D.M. is a Vanier scholar (CIHR). A.B. is also supported by an NSERC award, and L.P. by a CIHR award. We are grateful for platform support from CIHR, Genome Canada, Genome BC, Canada Foundation for Innovation and the Michael Smith Foundation for Health Research. The work was funded by the BC Cancer Foundation and the CBCF BC/Yukon chapter.

Author Contributions S.P.S. and R.D.M.: led the data analysis and wrote the manuscript. M.H.: oversaw the sequencing efforts. J.K., L.P., T.P., J.S., C.S., A.B., 
R.M. and T.S.: validation of variants. A.D.: primer design. K.G. and P.W.: establishment of TTR-BREAST tumour bank. K.T., R.G., R.A.H., S.J., M.S., G.L., A.E.T., R.V., G.A.T. and R.L.W.: bioinformatic analysis. G.T., D.H. and P.W.: sample selection and histological grading. Y.Z.: Illumina sequencing library preparation. C.C. and D.H.: data analysis and interpretation. S.A. and M.A.M.: conceived and oversaw the study and wrote the manuscript.
Author Information Genome sequence data have been deposited at the European Genotype Phenotype Archive (http://www.ebi.ac.uk/ega), which

is hosted by the EBI, under accession number EGAS00000000054. Reprints and permissions information is available at www.nature.com/reprints.

Correspondence and requests for materials should be addressed to M.A.M. (mmarra@bcgsc.ca) or S.A. (saparicio@bccrc.ca). 